\title{
Analysis of Clinicopathological Features of Cervical Mucinous Adenocarcinoma with a Solitary Ovarian Metastatic Mass as the First Manifestation
}

This article was published in the following Dove Press journal: Cancer Management and Research

\author{
Shuanghuan Liu (D) \\ Yunquan Guo 2,3 \\ Bin $\mathrm{Li}^{\prime}$ \\ Huijuan Zhang ${ }^{2}$ \\ Rong Zhang' \\ Shan Zheng ${ }^{2}$ \\ 'Department of Gynecologic Oncology, \\ National Cancer Center/National Clinical \\ Research Center for Cancer/Cancer \\ Hospital, Chinese Academy of Medical \\ Sciences and Peking Union Medical \\ College, Beijing, People's Republic of \\ China; ${ }^{2}$ Department of Pathology, \\ National Cancer Center/National Clinical \\ Research Center for Cancer/Cancer \\ Hospital, Chinese Academy of Medical \\ Sciences and Peking Union Medical \\ College, Beijing, People's Republic of \\ China; ${ }^{3}$ Department of Pathology, \\ Affiliated Tumor Hospital, Xinjiang \\ Medical University, Urumqi, People's \\ Republic of China
}

Correspondence: Bin Li

Department of Gynecologic Oncology,

National Cancer Center/National Clinical

Research Center for Cancer/Cancer

Hospital, Chinese Academy of Medical

Sciences and Peking Union Medical

College, No. 17, South Panjiayuan

Residential, Beijing, Chaoyang I0002I,

People's Republic of China

Tel/Fax +86-10-8778-8275

Email libin@cicams.ac.cn

\section{Shan Zheng}

Department of Pathology, National

Cancer Center/National Clinical Research

Center for Cancer/Cancer Hospital,

Chinese Academy of Medical Sciences and

Peking Union Medical College, No. 17,

South Panjiayuan Residential, Beijing,

Chaoyang I0002I,People's Republic of

China

Tel +86- $10-8778-75 \mid 4$

Fax +86-10-877I-8709

Email zhengshan@cicams.ac.cn
Purpose: To avoid misdiagnosis, clinicopathological features were analyzed in cases of cervical mucinous adenocarcinoma with solitary ovarian metastatic masses.

Patients and Methods: Three cases misdiagnosed as primary ovarian adenocarcinoma before surgery were filtered from the database of the Cancer Hospital/Chinese Academy of Medical Sciences from January 1998 to December 2016. The clinical data were thoroughly collected and compared, and both frozen and paraffin-embedded pathological sections were reviewed by two expert pathologists.

Results: None of the patients experienced cervical contact bleeding, and no typical cervical neoplasms were found. The cervical canals were slightly thickened in two patients, as detected by either palpation or imaging. The high-risk human papillomavirus (HPV) test results were all negative, and the thin-prep cytologic test (TCT) screened only one case of atypical glandular epithelial cells. All cases were indicative of higher serum CA19-9 levels (79.49-6124 U/mL). The ovarian masses showed no regular laterality, while they were all cystic or solid-cystic. Their pathological sections indicated a benign appearance of the capsule tissue with well-differentiated mucinous glands infiltrating the ovarian cortex with focal necrosis.

Conclusion: Attention should be paid to cervical examinations before and during surgery for cervical mucinous adenocarcinoma with a metastatic ovarian mass as the first manifestation. Such patients may gain a better prognosis after active treatment.

Keywords: cervical mucinous adenocarcinoma, metastatic ovarian mucinous adenocarcinoma, solitary ovarian metastasis, occult primary lesion, misdiagnosis, clinicopathological feature

\section{Introduction}

Cervical adenocarcinoma is a relatively common type of cervical cancer, accounting for $10-25 \%$ of its incidence. ${ }^{1}$ Arising from the cervical canal, the lesions are commonly endogenic and occult, and therefore, nearly $20 \%$ of patients lack clinical manifestations at the early stage. ${ }^{2}$ It has been reported that the rate of missed diagnosis with the thin-prep cytologic test (TCT) in patients with cervical adenocarcinoma is $34.8 \%,{ }^{3}$ and the negative rate with the high-risk human papillomavirus (HPV) test is $17.7 \%{ }^{4}$ which makes it difficult to detect the disease through screening in a timely fashion.

Ovarian metastases are rare in early cervical adenocarcinoma, and $8.98 \%$ of International Federation of Gynecology and Obstetrics (FIGO) stage IB-IIA 
cervical adenocarcinomas have been accompanied by ovarian metastases in the literature. ${ }^{3}$ Furthermore, cervical mucinous adenocarcinoma is one of the rare types of cervical adenocarcinoma, accounting for $18.6 \%$ of cervical adenocarcinoma cases. ${ }^{5}$ Ovarian metastasis rarely occurs in primary cervical mucinous adenocarcinoma, and the difficulty lies in the differential diagnosis from primary ovarian mucinous neoplasms. ${ }^{6}$

However, few authors have been able to incorporate any structured research into opinions related to improving diagnosis and selecting reasonable treatment for cervical mucinous adenocarcinoma with ovarian metastasis, especially with solitary metastasis lesions. To date, previous studies have focused mainly on case reports or pathological analyses. $^{7-9}$ This paper selected three cases of cervical mucinous adenocarcinoma with ovarian metastasis as the primary manifestation in one centre, and the clinicopathological features, treatment and prognosis were analysed in detail.

\section{Materials and Methods}

This study was approved by the ethics committee of the hospital, and written informed consent was obtained from every patient to publish the case details and any accompanying images (The 2017 Capital Clinical Characteristic Application Research Project supported by Beijing Municipal Science \& Technology Commission, P.R. China. No. NCC 2016 ST-05).

From January 1998 to December 2016, a total of 3 cases of ovarian metastasis from cervical mucinous adenocarcinoma were retrieved from the case database of the Cancer Hospital of the Chinese Academy of Medical Science. All of them presented with apparent ovarian masses as the first manifestation. The clinical manifestations, preoperative examination results, and surgical and postoperative treatment methods were analysed thoroughly, and all patients were followed until December 2019.

All three patients underwent rapid frozen pathological examination during the operation, and routine haematoxylin-eosin (HE) staining, pathological examination and immunohistochemical (IHC) staining were performed afterward. Two senior pathologists of the hospital were invited to review all of the pathological sections and analyse their pathological features.

\section{Results}

Clinical materials and pathological materials were included.
The general information of the three cases of secondary ovarian mucinous adenocarcinoma from the cervix with an ovarian mass as the first manifestation is shown in Table 1. The diagnostic and treatment courses are presented here:

Patient 1 was 49 years old with eumenorrhea, gravida-3 and para-2. She was admitted to the hospital for a "left ovarian mass found on physical examination". The TCT indicated atypical glandular cells from her previous physical examination report in another hospital, while both the re-examination of the TCT and the HPV test results were negative in our hospital. Pelvic magnetic resonance imaging (MRI) revealed a 0.5 -cm-thick cervical canal mucosa. Exploratory laparotomy was performed later. Approximately $300 \mathrm{~mL}$ mucous ascites was observed in the pelvic cavity during the operation. An isolated $6 \mathrm{~cm} \times 4.8 \mathrm{~cm}$ cystic mass was found on the left ovary, with a complete envelope and solid nodules on its surface. No other cancerous lesions were detected in the pelvic cavity during the comprehensive exploration. Upon gross examination of the left adnexa and tumour, it was found to be a cystic mass with solid nodules, presenting as a benign/borderline ovarian tumour; however, microscopically, welldifferentiated mucinous gonadal bodies infiltrated into the ovarian cortex, accompanied by focal necrosis and irregular morphology, which presented as a highly differentiated malignant tumour. Because of the inconsistent findings between the gross and microscopic appearance, the diagnosis pointed to metastatic mucinous carcinoma. The fact that the tumour nuclei were oval-shaped and lightly stained and that the chromatin was fine and uniform was different from the appearance of intestinal rod-shaped nuclei; therefore, an origin of the gastrointestinal tract was excluded. Based on the results of the preoperative gynaecological examination, the cervix was checked again under anaesthesia, a slightly thick and hard cervical canal was found, and cervical scraping and multipoint biopsy were performed. Fast frozen pathology indicated highly differentiated mucinous adenocarcinoma. Based on this, secondary ovarian mucinous adenocarcinoma from the cervix was diagnosed intraoperatively. Then, radical hysterectomy and comprehensive staging surgery were performed. Based on the paraffin pathology, the patient was diagnosed with stage IV cervical mucinous adenocarcinoma, and she received external pelvic radiation with intravenous chemotherapy. Recurrence had not occurred 99 months after the initial treatment.

Patient 2 was 49 years old, with gravida-4 and para-2 5 years after natural menopause. She was admitted to the hospital for "abdominal distension for 3 months". Both the TCT and cervical HPV test results were negative. A pelvic-enhanced 
Table I General Information of 3 Cases of Secondary Ovarian Mucinous Adenocarcinoma from Cervix with Ovarian Mass as the First Manifestation

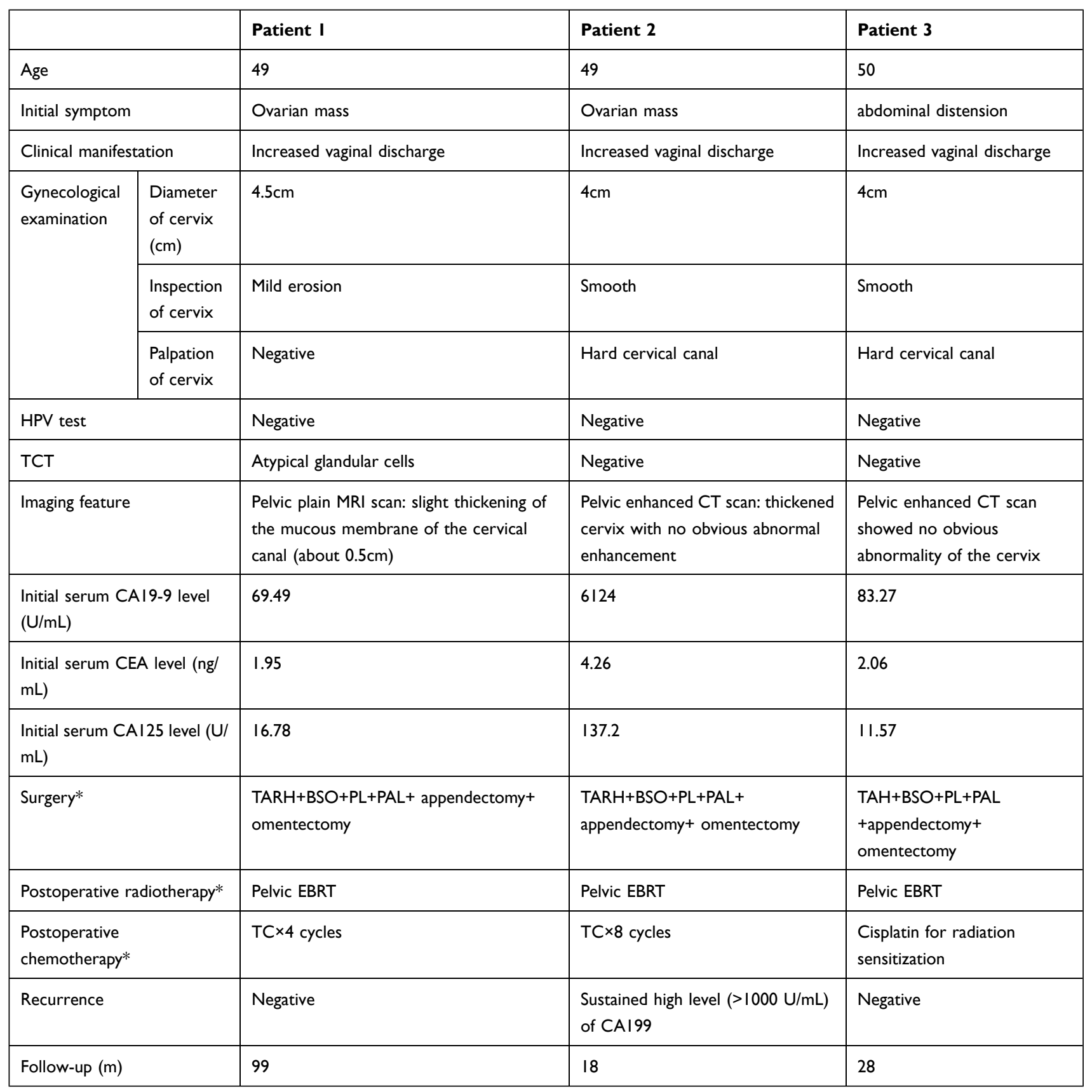

Note: * Specific treatment process.

Abbreviations: HPV, human papillomavirus; TCT, thin-prep cytologic test; CAI99, cancer antigen 199; CAI25, cancer antigen I25; CEA, carcinoma embryonic antigen; MRI, magnetic resonance imaging; CT, computed tomography; TARH, transabdominal radical hysterectomy; TAH, transabdominal hysterectomy; BSO, bilateral salpingooophorectomy; PL, pelvic lymphadenectomy; PAL, para-aortic lymphadenectomy; EBRT, external beam radiation therapy; TC, taxol+ carboplatin.

computerized tomography (CT) scan showed cervical enlargement without obvious abnormal enhancement. Laparotomy was performed, and approximately $500 \mathrm{~mL}$ of bloody ascites was observed in the pelvic cavity. There was one mass with a smooth surface and an intact capsule in each ovary, and the left and right masses were $10.3 \mathrm{~cm} \times 6.9 \mathrm{~cm}$ and
$11.5 \mathrm{~cm} \times 8.8 \mathrm{~cm}$ in size, respectively. The frozen pathology results showed that the bilateral masses were cystic and solid. Microscopically, well-differentiated mucinous gonadal bodies infiltrated into the ovarian cortex, accompanied by focal necrosis and irregular morphology, presenting as a highly differentiated malignant tumour. The above general findings were 
inconsistent with those seen under the microscope, and metastatic carcinoma was considered. Because of the different appearance of the tumour nuclei, an origin of the gastrointestinal tract was excluded. Then, cervical frozen biopsy was performed in the hard part of the cervical canal. Dramatically, highly differentiated mucinous adenocarcinoma was reported; therefore, secondary ovarian mucinous adenocarcinoma from the cervix was diagnosed intraoperatively. Then, the patient underwent radical hysterectomy and comprehensive staging surgery. Based on the paraffin pathology results, the patient was diagnosed with stage IV cervical mucinous adenocarcinoma, and then she received external pelvic radiation and intravenous chemotherapy. Recurrence had not occurred 18 months after the initial treatment.

Patient 3 was 50 years old, with gravida-3 and para-1 9 years after natural menopause. She was admitted to the hospital for a "left ovarian mass". Both the TCT and cervical HPV test results were negative. A pelvic-enhanced CT scan showed no significant abnormalities in the cervix. During laparotomy, no ascites were found, and no cytological abnormalities were reported in the pelvic cavity-flushing fluid. There was a solitary cystic mass $6.5 \mathrm{~cm} \times 4.5 \mathrm{~cm}$ in size with a complete surface on the left ovary. Frozen pathology sections suggested highly differentiated mucous carcinoma. Then, she directly underwent comprehensive staging surgery, including total hysterectomy. However, well-differentiated mucinous adenocarcinoma was observed in the cervical canal tissue. Based on the paraffin pathology results, the diagnosis was modified to stage IV cervical mucinous adenocarcinoma. External pelvic irradiation combined with cisplatin sensitization chemotherapy was applied after surgery. After 28 months of initial treatment, no recurrence was observed.

The general pathological information of the 3 cases is listed in Table 2. For the frozen pathological sections of the ovarian tumours, HE staining indicated welldifferentiated mucinous glands scattered in a multifocal distribution, accompanied by focal necrosis infiltrating into the normal ovarian cortex in the frozen sections (Figure 1). For the paraffin pathological sections, typical pathological manifestations of $\mathrm{HE}$ and IHC staining are shown in Table 3 and Figure 1.

\section{Discussion}

In this study, no cervical primary lesions were detected before surgery in all three cases. Several studies have pointed out that cervical mucinous adenocarcinoma probably has an insidious onset; however, some patients can

Table 2 General Pathological Information of 3 Cases of Secondary Ovarian Mucinous Adenocarcinoma from Cervix with Ovarian Mass as the First Manifestation

\begin{tabular}{|c|c|c|c|c|}
\hline & & Patient I & Patient 2 & Patient 3 \\
\hline \multirow{4}{*}{$\begin{array}{l}\text { General observation of } \\
\text { ovarian Mass }\end{array}$} & Laterality & Unilateral (Left side) & Bilateral & Unilateral (Left side) \\
\hline & Size $(\mathrm{cm})$ & $6 \times 4.8$ & $\begin{array}{l}\text { L } 10.3 \times 6.9 \\
\text { R II } 1.5 \times 8.8\end{array}$ & $6.5 \times 4.5$ \\
\hline & Character & Cystic & Cystic & Cystic \\
\hline & Other component & Solid nodules & Solid component in left mass & \\
\hline \multirow[t]{2}{*}{ Ascites } & Character & Viscous & Bloody & \\
\hline & Volume $(\mathrm{mL})$ & 300 & 100 & Negative \\
\hline \multirow[t]{5}{*}{ Cervical Cancer } & Size $(\mathrm{cm})$ & $2 * 0.8$ & $3 * 2$ & $2 * 1.2$ \\
\hline & Depth of invasion & $\geq 2 / 3$ & $\geq 2 / 3$ & $\geq 2 / 3$ \\
\hline & Invasion of parametrium & Negative & Negative & Negative \\
\hline & LVSI & Negative & Positive & Negative \\
\hline & Invasion of uterus & Negative & Lower uterine segment & Negative \\
\hline \multicolumn{2}{|l|}{ PLN metastasis } & Negative & Negative & Negative \\
\hline \multicolumn{2}{|l|}{ PALN metastasis } & Negative & Negative & Negative \\
\hline
\end{tabular}

Abbreviations: PLN, pelvic lymph node; PALN, para-aortic lymph node; LVSI, lymph vascular space invasion. 


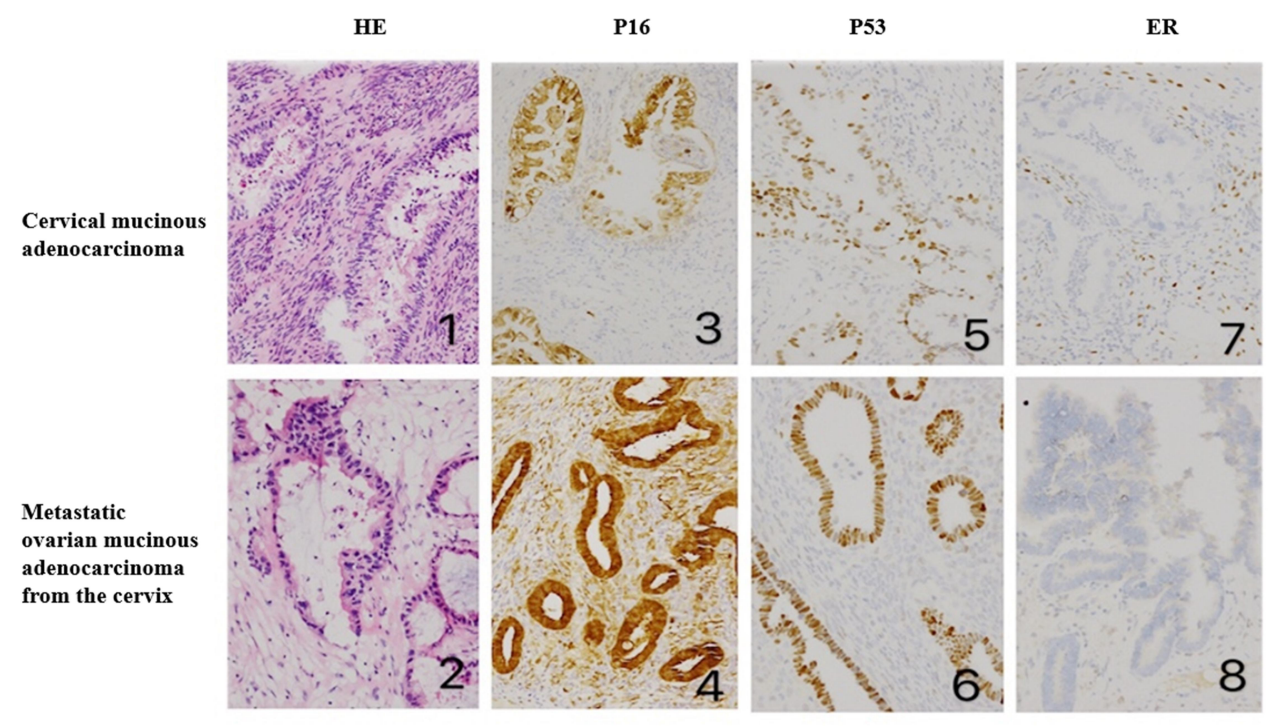

Figure I Typical pathological manifestations of primary cervical highly differentiated mucinous adenocarcinoma and metastatic ovarian highly differentiated mucinous adenocarcinoma (HE staining and IHC staining: 200X).

Notes: (I-I From Patient I) The frozen section of cervical mucinous adenocarcinoma showed a well-differentiated mucous gonadal body with focal epithelial atypia, nuclear enlargement, lamination, hyper-chromatin and necrosis in the glandular cavity. (I-2 From Patient I) Ovarian metastatic mucinous adenocarcinoma (cervical origin) indicated mild to moderate atypia of the mucous epithelium and obvious nuclear atypia. It was easy to confuse with primary ovarian myxoma according to the pathological morphology. (I-3 From Patient 2) Uniformly positive PI6 staining of cervical mucinous adenocarcinoma. (I-4 From Patient 2) Diffusely strong positive PI6 staining of metastatic ovarian mucinous adenocarcinoma from the cervix. (I-5 From Patient 2) Positive P53 staining of cervical mucinous adenocarcinoma. (I-6 From Patient 2) Positive P53 staining of metastatic ovarian mucinous adenocarcinoma from the cervix. (I-7 From Patient 3) Negative ER staining of cervical mucinous adenocarcinoma. (Figure I-8, Patient 3) Negative ER staining of metastatic ovarian mucinous adenocarcinoma from the cervix.

Abbreviations: HE, haematoxylin eosin; PI6, protein 16; P53, protein 53; ER, oestrogen receptor.

present with vaginal mucoid or watery secretion during the early stage, ${ }^{1,10}$ which can serve as a clue. In this study, all three patients presented with increased vaginal secretion to varying degrees. Clinical examination of the three patients showed thickened and/or hard cervical canals. This manifestation is consistent with the characteristics of cervical adenocarcinoma and should also cause suspicion. However, the patient's preoperative TCT and HPV screening results did not clearly indicate abnormalities, which led to the neglect of further examination of the cervical lesions. The cell structure of highly differentiated cervical mucous adenocarcinoma is similar to that of normal cervical canal epithelial cells, which is one of the main reasons for the low TCT recognition rate. ${ }^{11}$ In addition, all three patients in this study were in the perimenopausal period, and the migration of the transformed area also causes difficulties in sampling cervical cell specimens and HPV detection. ${ }^{12}$ As a consequence, falsenegative results on both the TCT and HPV test led to a missed diagnosis of primary cervical mucinous adenocarcinoma. Imaging also plays an important role in the diagnosis of cervical adenocarcinoma, especially in detecting lesions in the cervical canal, and MRI is superior to CT in this field. ${ }^{13}$ In this study, Patient 1 underwent a pelvic MRI plain scan before surgery, and a slight thickening of the cervical canal mucosa was found. The other two patients underwent a CT scan, one of which found cervical canal thickening, but no indication of a typical cervical tumour was provided in any of the three cases, which may be related to the fact that the ovarian tumours were the main focus of the doctors and the imaging examinations.

The existing prediction model can also assist in the differentiation of ovarian primary and metastatic mucinous adenocarcinoma. ${ }^{14-16}$ The model proposed by Seidman et $\mathrm{al}^{16}$ is currently the most widely used. It is believed that bilateral ovarian masses of any size or unilateral ovarian masses with diameters $\leq 10 \mathrm{~cm}$ are metastatic, while unilateral ovarian masses with diameters $>10 \mathrm{~cm}$ are primary mucinous adenocarcinomas. All three cases in this study are consistent with the prediction results of the model described above. Based on the indexes in the imaging examinations and the prediction model, the possibility of ovarian metastatic cancer in this study should have been taken into consideration. This model can aid in improving the screening of primary cervical lesions and reducing the chance of preoperative misdiagnosis. 
Table 3 Typical Pathological Manifestations of 3 Patients

\begin{tabular}{|c|c|c|c|c|c|}
\hline & & & Patient I & Patient 2 & Patient 3 \\
\hline \multirow[t]{3}{*}{$\begin{array}{l}\text { Cervical mucinous } \\
\text { adenocarcinoma }\end{array}$} & $\begin{array}{l}\text { Frozen } \\
\text { section }\end{array}$ & $\begin{array}{l}\mathrm{HE} \\
\text { staining }\end{array}$ & $\begin{array}{l}\text { Well-differentiated mucinous } \\
\text { glands infiltrating normal } \\
\text { mesenchyme with focal } \\
\text { necrosis }\end{array}$ & $\begin{array}{l}\text { Well-differentiated mucinous } \\
\text { glands infiltrating normal } \\
\text { mesenchyme with focal } \\
\text { necrosis }\end{array}$ & $\begin{array}{l}\text { Well-differentiated mucinous } \\
\text { glands infiltrating normal } \\
\text { mesenchyme with focal } \\
\text { necrosis }\end{array}$ \\
\hline & \multirow[t]{2}{*}{$\begin{array}{l}\text { Paraffin } \\
\text { section }\end{array}$} & $\begin{array}{l}\mathrm{HE} \\
\text { staining }\end{array}$ & $\begin{array}{l}\text { Well-differentiated mucinous } \\
\text { glands infiltrating normal } \\
\text { mesenchyme with focal } \\
\text { necrosis }\end{array}$ & $\begin{array}{l}\text { Well-differentiated mucinous } \\
\text { glands infiltrating normal } \\
\text { mesenchyme with focal } \\
\text { necrosis }\end{array}$ & $\begin{array}{l}\text { Well-differentiated mucinous } \\
\text { glands infiltrating normal } \\
\text { mesenchyme with focal } \\
\text { necrosis }\end{array}$ \\
\hline & & $\begin{array}{l}\mathrm{IHC} \\
\text { staining }\end{array}$ & $\begin{array}{l}\text { HR-HPV(-) PI6(++) P53(++) } \\
\operatorname{ER}(-) \\
\operatorname{CEA}(+)\end{array}$ & $\begin{array}{l}\text { HR-HPV(-) PI6(+++) P53(++) } \\
\text { ER(-) } \\
\text { CEA(-) }\end{array}$ & $\begin{array}{l}\text { HR HPV(-) PI6(+++) P53(++) } \\
\text { ER(-) CEA(-) CK7(+) CAI99(+ } \\
+) \text { CAI } 25(-)\end{array}$ \\
\hline \multirow[t]{3}{*}{$\begin{array}{l}\text { Metastatic mucinous } \\
\text { adenocarcinoma of } \\
\text { the ovary }\end{array}$} & $\begin{array}{l}\text { Frozen } \\
\text { section }\end{array}$ & & $\begin{array}{l}\text { Capsule tissue showed benign } \\
\text { appearance with well- } \\
\text { differentiated mucinous glands } \\
\text { infiltrating ovarian cortex with } \\
\text { focal necrosis }\end{array}$ & $\begin{array}{l}\text { Capsule tissue showed benign } \\
\text { appearance with well- } \\
\text { differentiated mucinous glands } \\
\text { infiltrating ovarian cortex with } \\
\text { focal necrosis }\end{array}$ & $\begin{array}{l}\text { Capsule tissue showed benign } \\
\text { appearance with well- } \\
\text { differentiated mucinous glands } \\
\text { infiltrating ovarian cortex with } \\
\text { focal necrosis }\end{array}$ \\
\hline & \multirow[t]{2}{*}{$\begin{array}{l}\text { Paraffin } \\
\text { section }\end{array}$} & $\begin{array}{l}\mathrm{HE} \\
\text { staining }\end{array}$ & $\begin{array}{l}\text { Capsule tissue showed benign } \\
\text { appearance with well- } \\
\text { differentiated mucinous glands } \\
\text { infiltrating ovarian medulla } \\
\text { cortex with focal necrosis }\end{array}$ & $\begin{array}{l}\text { Capsule tissue showed benign } \\
\text { appearance with well- } \\
\text { differentiated mucinous glands } \\
\text { infiltrating ovarian medulla } \\
\text { cortex with focal necrosis }\end{array}$ & $\begin{array}{l}\text { Capsule tissue showed benign } \\
\text { appearance with well- } \\
\text { differentiated mucinous glands } \\
\text { infiltrating ovarian medulla } \\
\text { cortex with focal necrosis }\end{array}$ \\
\hline & & $\begin{array}{l}\mathrm{IHC} \\
\text { staining }\end{array}$ & $\begin{array}{l}\text { HR-HPV(-) PI6(++) P53(++) } \\
\text { ER(-) } \\
\text { CEA }(+)\end{array}$ & $\begin{array}{l}\text { HR-HPV(-) PI6(+++) P53(++) } \\
\text { ER(-) } \\
\text { CEA(-) }\end{array}$ & $\begin{array}{l}\text { HR-HPV(-) PI6(++) P53(++) } \\
\text { ER(-) } \\
\text { CEA(-) CK7(++) CAI99(++) } \\
\text { CAI25(-) }\end{array}$ \\
\hline
\end{tabular}

Abbreviations: HE, hematein eosin; IHC, immunohistochemistry; HR-HPV, high-risk human papillomavirus; ER, estrogen receptor; PR, progesterone receptor; CK, cytokeratin; PI6, protein 16; P53, protein 53; CEA, carcinoma embryonic antigen; CA199, cancer antigen 199; CAI25, cancer antigen I25.

In addition, cervical mucinous adenocarcinoma can present with elevated serum levels of the tumour marker CA199 and normal levels of CA125 and CEA, ${ }^{17}$ while serum CEA is highly expressed in primary ovarian or gastrointestinal metastatic mucinous adenocarcinoma. ${ }^{18}$ In this study, all three patients showed a significant increase in CA199 levels and a normal level of CEA, which can also be used for identification.

Metastatic mucinous adenocarcinoma of the ovary accounts for $77 \%$ of cases of mucinous adenocarcinoma of the ovary. ${ }^{16}$ Tumours usually have the following characteristics: generally bilateral ovarian masses or small unilateral tumours (usually less than $10 \mathrm{~cm}$ in diameter). Microscopically, the tumour involves the ovarian surface and/or superficial cortex, presenting with an infiltrating growth pattern with multifocal or nodular distribution, surrounding the normal ovarian tissue with an interstitial proliferative response and obvious abnormal angiogenesis.
This type of tumour is obviously different from primary ovarian mucinous adenocarcinoma. ${ }^{16,19}$ In this study, one case (Patient 2) demonstrated bilateral small lesions, and one case (Patient 1) was unilateral. The general performance of these two cases was consistent with the above pathological characteristics. During the intraoperative pathological process, an origin of the gastrointestinal tract was excluded in these two cases because the tumour nuclei were ovoid and morphologically different from the intestinal rod-shaped nuclei. Combined with further exploration of the cervix, cervical biopsy was performed on each patient, and the diagnosis was confirmed in a timely fashion. Additionally, there was another patient (Patient 3) who was not correctly diagnosed during the operation. She was judged as having ovarian highly differentiated mucous carcinoma by frozen pathology. Previous studies have indicated that metastatic ovarian mucinous adenocarcinoma from the cervix is prone to mimicking 
primary ovarian mucinous tumours in terms of the pathological manifestations, such as a generally large unilateral mass with a smooth surface and multicavity separation and microscopically well-differentiated ethmoidal, villous or papillary glands infiltrating the normal cortex. , $^{9,21}$ Reviewing the pathological sections, Patient 3 demonstrated the characteristics described above. Therefore, it is very difficult to determine the cervical origin of ovarian tumours through intraoperative frozen pathology alone. More attention should be paid to strengthening the preoperative detection of cervical lesions and combining this detection with pathological examinations.

The pathological comparison between the ovary and cervix after surgery is the key to final diagnosis, in which immunohistochemistry plays an important role. Studies have reported that ovarian mucinous adenocarcinoma of cervical origin commonly shows oestrogen receptor (ER), progesterone receptor (PR) and cytokeratin 20 (CK20) negatively but protein 53 (p53) and cytokeratin 7 (CK7) positivity. These manifestations are often contrary to those of primary ovarian neoplasms. ${ }^{10,22}$ It is worth noting that even with HPV-negative tumors, nearly $50 \%$ of cervical mucinous adenocarcinomas still demonstrate protein 16 (p16) positivity, which can be used as an important clue for determining a cervical origin of ovarian metastatic tumours. ${ }^{1,10,23,24}$ In this study, tumours from both the cervix and the ovary in the three patients all showed negative HPV results but positive p16 results according to IHC staining, which verified the diagnosis of ovarian metastasis of primary mucinous adenocarcinoma from the cervix. The result of HPV negativity with p16 positivity may be less representative due to the small number of rare cases, and the absence of HPV positivity could be related to different detection sensitivities or the inability of detection for rare HPV subtypes.

It has been reported that synchronous gynaecological malignancies account for up to $2 \%$ of women diagnosed with any kind of gynaecological cancer, and the most common association consists of ovarian and endometrial cancer. For synchronous ovarian and cervical primary cancers, isolated cases have rarely been reported. ${ }^{23}$ The most common association is HPV-related cervical cancer and mucinous ovarian adenocarcinoma, which is different from the cases in this study. Different situations of HPV infection and distinct histological types among the cervix and ovary are conducive to the final diagnosis. ${ }^{24}$ The cases in this study also require differential diagnosis with minimal deviation adenocarcinoma (MDA)/adenoma malignum, which is commonly unrelated to HPV and easily missed on cytology. Profuse vaginal discharge is considered to be a suspicious presentation. Firm and white/tan/yellow in colour, these tumours can form a "barrel-shaped" cervix, in addition to polypoid and ulcerative forms. Because of the precursor lesion called lobular endocervical glandular hyperplasia, multilobular cysts at the uterine cervix may be detected by ultrasonography. Microscopically, MDA is characterized by benign-appearing, irregularly shaped glands that often deeply infiltrate the cervical stroma, sometimes with neurovascular invasion. The tumour cells usually contain abundant clear, foamy or pale eosinophilic cytoplasm with generally low nuclear atypia and nuclear-cytoplasmic ratios. They may indicate typical intestinal differentiation in the form of goblet cells and neuroendocrine-like eosinophilic granular cytoplasm. MDA has variable positivity for CEA, p53, MUC6, HIK1083, SMA, CA125 and Ki67, which is helpful for further diagnosis. In addition, MDA may coexist with Peutz-Jeghers syndrome, which can serve as a clue. In this study, no typical abnormalities, such as a "barrel-shaped" cervix or multilobular cysts, were found, and no obvious malignant biological behaviours were seen pathologically. Immunohistochemistry also indicated negative results for specific markers of MDA. In conclusion, the cases in this study were not considered MDA.

Sakuragi $^{23}$ found that the rate of ovarian metastasis in patients with positive vascular invasion of cervical cancer was significantly higher than that of patients with negative invasion. Based on this hypothesis, haematologic metastasis may be the main route for ovarian metastasis of cervical cancer. Shimada et $\mathrm{al}^{24}$ believed that cervical adenocarcinoma was transferred to the ovary mainly by blood vessels. The tumour may metastasize to the ovaries by invading the myometrium of the cervix and the lower uterine segment, spreading to the vascular plexus in the parametrium and transferring to the ovary. None of the three patients in this study showed parametrium infiltration or lymph node metastasis, but deep interstitial infiltration of the cervix appeared at the same time. As a result, the possibility of haematogenous metastasis to the ovary was determined in these three patients. Based on this, the treatment of relatively isolated ovarian metastasis from the cervix was explored and discussed.

Generally, the overall prognosis of stage IV cervical mucinous adenocarcinoma is poor, and the treatment of cervical mucinous adenocarcinoma with ovarian metastasis has only been reported in individual cases. Sun et $\mathrm{al}^{8}$ reported a case of ovarian metastasis of stage IV cervical mucinous adenocarcinoma. The patient also had a complication of 
pelvic peritoneal planting. Due to misdiagnosis as a primary ovarian neoplasm, cytoreduction surgery was performed, followed by postoperative chemotherapy. The patient gained a progression-free survival of 15 months. Khor et $\mathrm{al}^{7}$ reported a case of primary cervical mucinous adenocarcinoma with isolated ovarian metastasis. With complete surgical staging and consolidation radiotherapy, the patient achieved an overall survival of 45 months. In this study, three patients underwent comprehensive staging surgery and postoperative chemoradiotherapy, and no recurrence occurred during the follow-up period of 18-99 months. These results suggest that although isolated ovarian metastasis is referred to as stage IV, it may obtain a good prognosis from active treatment. In this regard, Nagel et $\mathrm{a}^{25}$ believed that resection of ovarian metastatic lesions is one of the essential elements to the survival of patients because of tumour-load reduction. However, the method of hysterectomy is still controversial. In this study, two patients were correctly diagnosed during the operation, so radical hysterectomy was performed. The authors believe that the implementation of radical hysterectomy may be a reasonable approach to achieve a better prognosis for patients with cervical mucinous adenocarcinoma and solitary ovarian metastasis.

The weakness of this study is its retrospective nature and its small sample size. However, it does provide a relatively systematic analysis of this rare case. Comprehensive studies in larger populations and multiple centres are needed to further verify the unknown factors, such as improving the diagnosis and forming a standardized treatment protocol for cervical mucinous adenocarcinoma with isolated ovarian metastasis.

\section{Conclusion}

Cervical mucinous adenocarcinoma with an ovarian mass as the first manifestation is easily misdiagnosed before surgery. Mucinous cervical adenocarcinoma with solitary ovarian metastasis may have a better prognosis with active treatment.

\section{Disclosure}

The authors report no conflicts of interest in this work.

\section{References}

1. Park KJ. Cervical adenocarcinoma: integration of HPV status, pattern of invasion, morphology and molecular markers into classification. Histopathology. 2020;76(1):112-127. doi:10.1111/his.13995

2. Marinova P, Rampalova G, Kolnikova S, Orthova S, Ondriasch F. Endocervical adenocarcinoma-a current diagnostic problem. Akusherstvo I Ginekologiia. 2010;49(7):35-41.
3. Nakanishi T, Wakai K, Ishikawa H, et al. A comparison of ovarian metastasis between squamous cell carcinoma and adenocarcinoma of the uterine cervix. Gynecol Oncol. 2001;82(3):504-509. doi:10.1006/ gyno.2001.6316

4. Tao X, Zheng B, Yin F, et al. Polymerase Chain Reaction Human Papillomavirus (HPV) detection and HPV genotyping in invasive cervical cancers with prior negative $\mathrm{HC} 2$ test results. Am J Clin Pathol. 2017;147(5):477-483.

5. Stolnicu S, Barsan I, Hoang L, et al. International Endocervical Adenocarcinoma Criteria and Classification (IECC): a new pathogenetic classification for invasive adenocarcinomas of the endocervix. Am J Surg Pathol. 2018;42(2):214-226. doi:10.1097/PAS.0000 000000000986

6. Makris GM, Chrelias C, Papanota A, Battista MJ, Papantoniou N. Ovarian tumors: should the cervix be examined first? Eur J Gynaecol Oncol. 2017;38(3):462-464.

7. Khor TS, Brennan BA, Leung YC, Sengupta S, Stewart CJ. Cervical adenocarcinoma metastatic to the ovary mimicking primary ovarian carcinoma. Pathology. 2009;41(4):397-400. doi:10.1080/003130209 02886993

8. Sun HD, Hsiao SM, Chen YJ, Wen KC, Li YT, Wang PH. Advanced endocervical adenocarcinoma metastatic to the ovary presenting as primary ovarian cancer. Taiwan J Obstet Gynecol. 2015;54 (2):201-203. doi:10.1016/j.tjog.2014.10.005

9. Ronnett. Endocervical adenocarcinomas with ovarian metastases. Am J Surg Pathol. 2008;32(12):1835-1853. doi:10.1097/PAS.0b013e 3181758831

10. Stolnicu S, Hoang L, Soslow RA. Recent advances in invasive adenocarcinoma of the cervix. Virchows Arch. 2019;475 (5):537-549. doi:10.1007/s00428-019-02601-0

11. Al-Nourhji O, Dermawan JK, Booth CN, Underwood D, AbdulKarim FW. Role of ThinPrep liquid-based cytology in evaluation of the endocervical canal in patients with abnormal cervical screening. J Am Soc Cytopathol. 2019;8(5):278-283. doi:10.1016/j.jasc.2019. 05.001

12. Conrad R, Wentzensen N, Zhang RR, et al. Distribution of cell types differs in Papanicolaou tests of squamous cell carcinomas and adenocarcinomas. $J$ Am Soc Cytopathol. 2017;6(1):10-15. doi:10.1016/j.jasc.2016.08.003

13. Saida T, Sakata A, Tanaka YO, et al. Clinical and MRI characteristics of uterine cervical adenocarcinoma: its variants and mimics. Korean J Radiol. 2019;20(3):364-377. doi:10.3348/kjr.2018.0458

14. Jung ES, Bae JH, Lee A, Choi YJ, Park JS, Lee KY. Mucinous adenocarcinoma involving the ovary: comparative evaluation of the classification algorithms using tumor size and laterality. $J$ Korean Med Sci. 2010;25(2):220-225. doi:10.3346/jkms.2010.25.2.220

15. Maeda-Taniguchi M, Ueda Y, Miyake T, et al. Metastatic mucinous adenocarcinoma of the ovary is characterized by advanced patient age, small tumor size, and elevated serum CA125. Gynecol Obstet Invest. 2011;72(3):196-202. doi:10.1159/000323962

16. Seidman JD, Kurman RJ, Ronnett BM. Primary and metastatic mucinous adenocarcinomas in the ovaries: incidence in routine practice with a new approach to improve intraoperative diagnosis. Am J Surg Pathol. 2003;27(7):985-993. doi:10.1097/00000478-20030700000014

17. Abiko K, Baba T, Ogawa M, et al. Minimal deviation mucinous adenocarcinoma ('adenoma malignum') of the uterine corpus. Pathol Int. 2010;60(1):42-47. doi:10.1111/j.1440-1827.2009.02473.x

18. Frumovitz M, Schmeler KM, Malpica A, Sood AK, Gershenson DM. Unmasking the complexities of mucinous ovarian carcinoma. Gynecol Oncol. 2010;117(3):491-496. doi:10.1016/j.ygyno.2010. 02.010

19. Kubeček O, Laco J, Špaček J, et al. The pathogenesis, diagnosis, and management of metastatic tumors to the ovary: a comprehensive review. Clin Exp Metastasis. 2017;34(5):295-307. doi:10.1007/ s10585-017-9856-8 
20. Reyes C, Murali R, Park KJ. Secondary involvement of the adnexa and uterine corpus by carcinomas of the uterine cervix: a detailed morphologic description. Int J Gynecol Pathol. 2015;34(6):551-563. doi:10.1097/PGP.0000000000000206

21. Casey L, Singh N. Metastases to the ovary arising from endometrial, cervical and fallopian tube cancer: recent advances. Histopathology. 2020;76(1):37-51. doi:10.1111/his. 13985

22. Stolnicu S, Barsan I, Hoang L, et al. Diagnostic algorithmic proposal based on comprehensive immunohistochemical evaluation of 297 invasive endocervical adenocarcinomas. Am J Surg Pathol. 2018;42(8):989-1000. doi:10.1097/PAS.0000000000001 090
23. Sakuragi N. A multivariate analysis of blood vessel and lymph vessel invasion as predictors of ovarian and lymph node metastases in patients with cervical carcinoma. Cancer. 2000;88(11):2578-2583. doi:10.1002/ 1097-0142(20000601)88:11<2578::AID-CNCR21>3.0.CO;2-Y

24. Shimada M, Kigawa J, Nishimura R, et al. Ovarian metastasis in carcinoma of the uterine cervix. Gynecol Oncol. 2006;101 (2):234-237. doi:10.1016/j.ygyno.2005.10.004

25. Nagel CI, Thomas SK, Richardson DL, Kehoe SM, Miller DS, Lea JS. Adnexal masses requiring surgical intervention in women with advanced cervical cancer. Gynecol Oncol. 2014;134(3):552-555. doi:10.1016/j.ygyno.2014.06.032

\section{Publish your work in this journal}

Cancer Management and Research is an international, peer-reviewed open access journal focusing on cancer research and the optimal use of preventative and integrated treatment interventions to achieve improved outcomes, enhanced survival and quality of life for the cancer patient.
The manuscript management system is completely online and includes a very quick and fair peer-review system, which is all easy to use. Visit http://www.dovepress.com/testimonials.php to read real quotes from published authors. 